\title{
Study on the Stability of Carotenoid Pigments
}

\author{
Luoyun Zheng, Zhengyang Wang, *Jiaying Xin, \\ Shengbo Gao and Tingting Yao \\ Key Laboratory for Food Science and Engineering \\ Harbin University of Commerce, Harbin, China \\ 506637547@qq.com, 306224963@qq.com, xinjiayingvip@163.com, \\ 544546692@qq.com,657407999@qq.com
}

Keywords: Rhodotorula; Carotenoids; Light; Heat; Acids; Oxidant agent; Reducing agent; Stability

\begin{abstract}
The effect of light, heat, acids, oxidant and reducing agent on the stability of carotenoids from Rhodotorula were studied, The results showed that the carotenoids from Rhodotorula are sensitive to heat, light, acid and oxidant . The absorbance at $475 \mathrm{~nm}$ of carotenoids was weakened with the raise of temperature. Carotenoids was unstable under light especially outdoor sunlight. Carotenoids were also unstable under ultraviolet and indoor scattering. The absorbance at $475 \mathrm{~nm}$ of carotenoids is almost no change in light-free condition. It has been found that the carotenoids from Rhodotorula were unstable under acidic condition. Oxidant (hydrogen peroxide) can also oxidized the carotenoids from Rhodotorula. However, Reducing agent (Vc) has protective effect on the carotenoids from Rhodotorula.
\end{abstract}

\section{Introduction}

Carotenoids are an important group of natural pigments with specific applications as colourants, feed supplements, nutraceuticals, medicine, cosmetic and biotechnological purposes [1]. They are precursors of vitamin A and are thus used for modifying the color of fats, oils, cheese and drinks [2]. Carotenoids are roughly classified into two groups. One is the hydrocarbon carotenes such as $\beta$ carotene, torulene, and the other is the oxygenated xanthophylls such as torularhodin and astaxanthin [3]. Astaxanthin has several essential biological functions including protection against UV light effects; immune response; pigmentation; communication; reproductive behavior and improved reproduction [4]. The major market for astaxanthin is as a pigmentation source in aquaculture, primarily in salmon and trout [5]. $\beta$-Carotene is an orange-yellow pigment of carotenoid family. It has been demonstrated that $\beta$-Carotene has anticancer and antioxidant properties [6]. Carotenoids can also serve as light quenchers and play an important role in photo protection [7]. The fruits can display different colour phenotypes, from orange to orange-red, depending on the lycopene/betacarotene ratio [8]. Carotenoids are significant industrially pigments which can be produced by many bacteria, fungi, and plants. It biosynthesis in yeasts is involved in stress response mechanisms. Thus, the controlled physiological and nutrition stress can be used for enhanced pigment production [9]. In this experiment, the effect of light, heat, acids, oxidant and reducing agent on the stability of carotenoids were studied to understand the physical and chemical properties of carotenoids from Rhodotorula [10].

\section{Materials and Methods}

The Extraction of Carotenoids. To take $500 \mathrm{ml}$ fermentation liquor, after centrifugation the supernatant was discarded, the wet cells were blanched into $3 \mathrm{~mol} / \mathrm{HCl}$ solution at $28^{\circ} \mathrm{C}$, and boiled in water for 4 - 5min and rapid cooled, and then centrifuged at 8,000 rpm for $15 \mathrm{~min}$. The supernatant was discarded and then acetone was added into the broken cells. The broken cells were washed 2 times by distilled water. Thereafter, extraction of carotenoids was carried out under the 
condition of $100 \mathrm{rpm}$ for $15 \mathrm{~min}$ at $28^{\circ} \mathrm{C}$. The supernatant was obtained by centrifugation $(8,000 \mathrm{rpm}$, 15min)

The Effect of Temperature on the Stability of Rhodotorula Pigment. In the condition of PH 6.0, the tube was wrapped with black paper that make it in the light-free condition. The extraction of carotenoids from Rhodotorula was incubated at $20{ }^{\circ} \mathrm{C} 30{ }^{\circ} \mathrm{C}, 40{ }^{\circ} \mathrm{C}, 50{ }^{\circ} \mathrm{C}, 60{ }^{\circ} \mathrm{C}$ respectively. The absorbance at $475 \mathrm{~nm}$ was determined periodically to analyze the effects of temperature on the stability of the Rhodotorula pigment.

The Effect of Light on the Stability of Rhodotorula Pigment. Pigment extraction of Rhodotorula was placed under the condition of light-free, indoor scattering light, outdoor sunlight and ultraviolet light respectively at $30{ }^{\circ} \mathrm{C}$ and $\mathrm{PH}$ 6.0. The absorbance at $475 \mathrm{~nm}$ was determined periodically to analyze the effects of light on the stability of the Rhodotorula pigment.

The Effect of Acid on the Stability of Rhodotorula Pigment. Pigment extraction of Rhodotorula was placed at $30{ }^{\circ} \mathrm{C}$ under the condition of light-free with different concentration of acetic acid. The absorbance at $475 \mathrm{~nm}$ was determined periodically to analyze the effects of acid on the stability of the Rhodotorula pigment.

The Effect of Oxidants on the Stability of Rhodotorula Pigment. Pigment extraction of Rhodotorula was placed at $30{ }^{\circ} \mathrm{C}$ under the condition of light-free and different concentration of hydrogen peroxide. The absorbance at $475 \mathrm{~nm}$ was determined periodically to analyze the effects of oxidants on the stability of the Rhodotorula pigment.

The Effect of Reducing Agents on the Stability of Rhodotorula Pigment. Pigment extraction of Rhodotorula was placed at $30{ }^{\circ} \mathrm{C}$ under the condition of light-free and different concentration of Vc. The absorbance at $475 \mathrm{~nm}$ was determined periodically to analyze the effects of reducing agents on the stability of the Rhodotorula pigment.

\section{Results}

Effect of Temperature on Carotenoids Stability. As shown in Fig. 1, when the temperature was lower than $40{ }^{\circ} \mathrm{C}$, the absorbance at $475 \mathrm{~nm}$ of the Rhodotorula pigment was almost no change during $3 \mathrm{~h}$ incubation. This indicated that the Rhodotorula pigment was stable at temperature no more than $40{ }^{\circ} \mathrm{C}$. When the temperature is $50{ }^{\circ} \mathrm{C}$ and $60{ }^{\circ} \mathrm{C}$, the absorbance at $475 \mathrm{~nm}$ of the Rhodotorula pigment was decreased during $3 \mathrm{~h}$ incubation. Therefore, when the temperature rises to above $40{ }^{\circ} \mathrm{C}$, it will affect the stability of the Rhodotorula pigment.

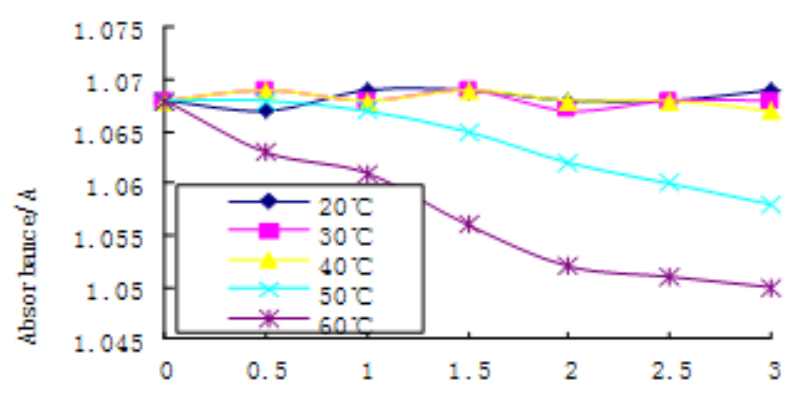

Figure 1. The effect of tempera ture 


\section{Effect of Light on Carotenoids Stability.}

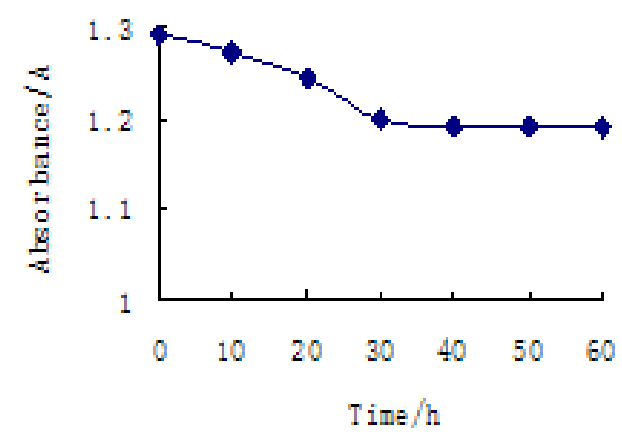

Figure 2. Effect of ultraviolet light

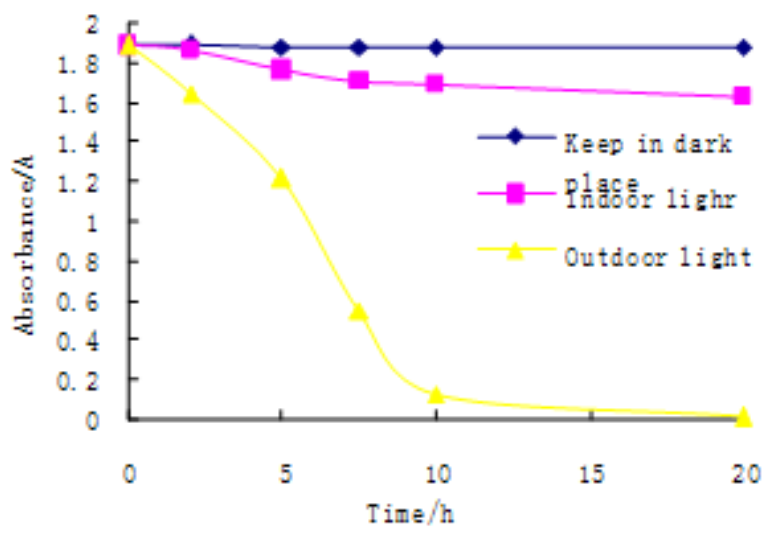

Figure 3. Effect of different absorbance

As shown in Fig. 2 and Fig. 3, the Rhodotorula pigment is unstable to UV light and outdoor light.Light is a significant factor that can decrease the stability of Rhodotorula pigment; especially for Outdoor light. The absorbance values of Rhodotorula pigment was decreased substantially with the increase of exposure time under Outdoor lingt. The absorbance values of Rhodotorula pigment was decreased to zero in a very short time, The UV, indoor light and Keep in dark also have an impact on the stability of rhodotorula pigment.

\section{Effect of Acid on Carotenoids Stability}

Fig. 4 showed the effect of acid on carotenoids stability

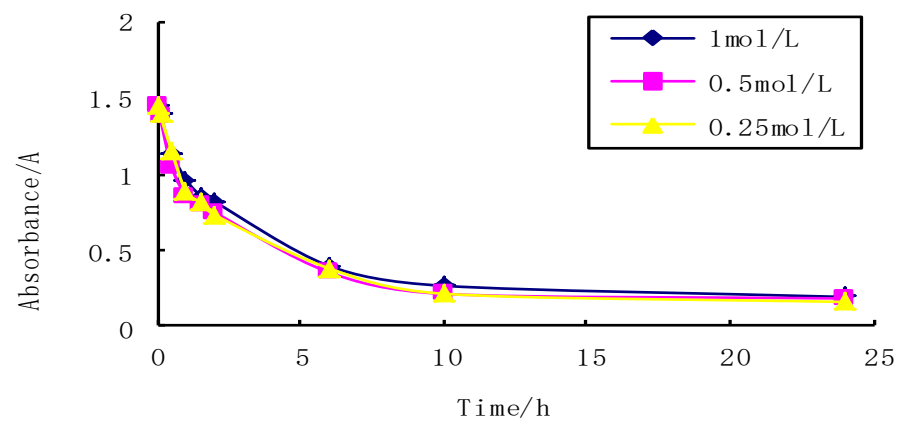

Figure 4. Effect of acid(acetic acid)

Acid have a certain impact on the stability of the carotenoids. As shown in Fig. 4. After the addition of acetic acid, a larger decrease in absorbance of Rhodotorula pigment within 10 hours has been found. However, different concentrations of acetic acid had no significant effect on the results in this experiment. 
Effect of Oxidants on Carotenoids Stability. Fig. 5 showed the effect of oxidants on the stability of carotenoids from Rhodotorula

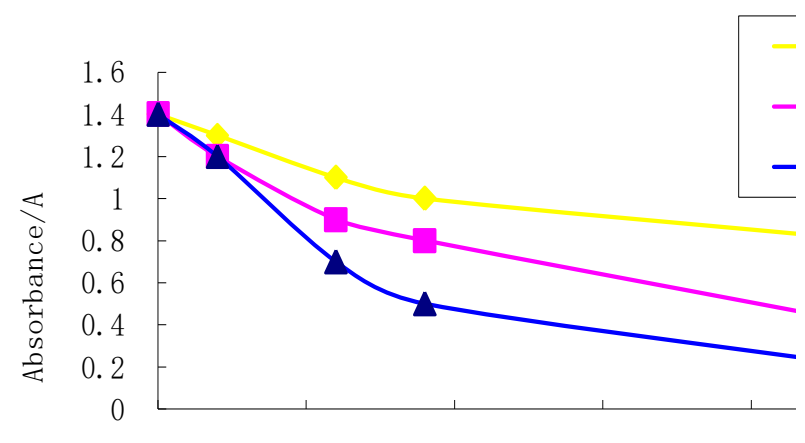

Figure 5 Effect of oxidant $\left(\mathrm{H}_{2} \mathrm{O}_{2}\right)$

Oxidant is a greater impact factor on the stability of rhodotorula pigment. After adding an oxidizing agent, The absorbance values of Rhodotorula pigment was decreased rapidly

Effect of Reducing Agents on Carotenoids Stability. Fig. 6: showed the effect of reducing agents on the stability of carotenoids from Rhodotorula

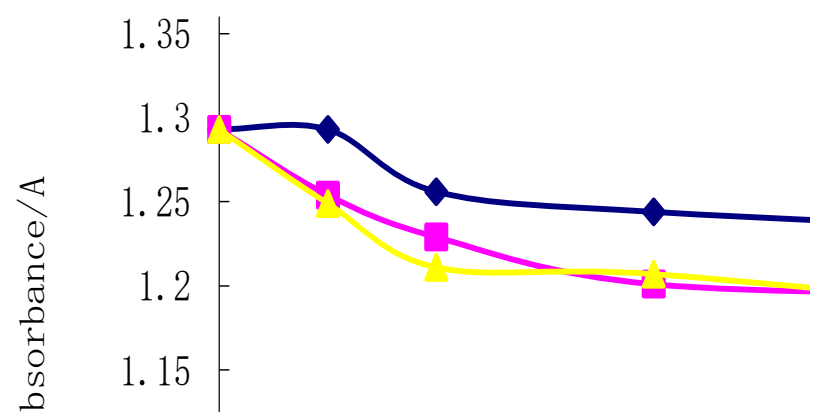

Figure 6 Effect of reducing agent $\left(\mathrm{V}_{\mathrm{c}}\right)$

As shown in Fig. 6, reducing agents has a good protective effect on rhodotorula pigment. After adding reducing agent, the absorbance values of Rhodotorula pigment did not change significantly with the increase of storage time.

\section{Conclusion}

The rhodotorula carotenoids is unstable to temperature.. it is sensitive and very unstable on outdoor light especially sunlight. Also, the rhodotorula carotenoids is acid unstable. Oxidant (hydrogen peroxide) can oxidize the carotenoids from Rhodotorula. However, Reducing agent (Vc) has protective effect on the carotenoids from Rhodotorula.

\section{Refrences}

[1] Choudhari Sheetal M, Ananthanarayan Laxmi, Singhal Rekha S. "Use of metabolic stimulators and inhibitors for enhanced production of beta-carotene and lycopene by Blakeslea trispora", Bioresource Technology, Vol.2008,Vol.16 July 2008 ,doi:10.1016/j.biortech. 2007.05.051 pp. 2895 - 2896.

[2] Edge, R, McGarvey, D. J. and Truscott, T. G., "The Carotenoids as Antioxidants- a Review," New Trends in , Vol.21 Augest1997 pp.189 -200 
[3] Young, A. and Britton, G., "Carotenoids in Photosynthesis,"M Chapman \& Hall, London, UK (1993)

[4] Martin Guerin, Mark E. Huntley and Miguel Olaizola, "Haematococcus astaxanthin: applications for human health and nutrition," TRENDS in Biotechnology, Vol.21, May 2003, pp.210-216, doi:10.1016/S0167-7799(03)00078-7.

[5] R. Todd Lorenz and Gerald R. Cysewski, "Commercial potential for Haematococcus microalgae as a natural source of astaxanthin", TIBTECH APRIL, Vol. 18, 2000, pp.160-167

[6] Astorg, P, " Food carotenoids and cancer prevention: an overview of current research," Trends Food in Food Science and]Technology, Vol.8, December 1997, pp. 406-412

[7] Phaff HJ, Fell JW (1970) Chapter 8, Discussion of the genera of asporogenous yeasts not belonging to the sporobolomycetaceae Genus 3, In: Lodder J (ed) The yeasts-a taxonomic study.North-Holland, Amsterdam, pp. 1088-1145

[8] Ermakov Igor V, Ermakova Maia R, Gellermann Werner et al. "Noninvasive selective detection of lycopene and beta-carotene in human skin using Raman spectroscopy,". Biomedical Optics”, Vol .9 May/April 2004 No.2

[9] Marova I, Carnecka M, Halienova A et al, "Use of several waste substrates for carotenoid-rich yeast biomass production”.Environmental Management , Vol.95,2012,pp.s338 -s342, doi:10.1016/j.jenvman.2011.06.018

[10]Ginka I. Frengova - Dora M. Beshkova. "Carotenoids from Rhodotorula and PhaYa:yeasts of biotechnological importance”, Ind Microbiol Biotechnol,Vol.2009 No.36 ,pp.163-164 